\title{
Corrosion Behavior of Stainless Steel 304 in Formic Acid Solution
}

\author{
Isao Sekine* and Akira Chinda* \\ *Department of Industrial Chemistry, Faculty of Science and Technology, \\ Science University of Tokyo
}

\begin{abstract}
The corrosion behavior of Type 304 SS in formic acid was investigated by measuring the corrosion weight loss, the polarization curve and the impedance at the steel-solution interface. The results are summarized as follows. (1) The corrosion rate of the steel was found to change with the remarkable dependence on the concentration of formic acid and the temperature. (2) The corrosion potential $\left(E_{\mathrm{corr}}\right)$ showed a linear relationship against $\mathrm{pH}$ of formic acid solution, and the cathodic reaction was approximately first order with respect to the activity of proton. (3) The selective corrosion dissolution of $\mathrm{Fe}, \mathrm{Cr}$ and $\mathrm{Ni}$ of this steel was not observed.
\end{abstract}

\section{Introduction}

The study of corrosion behavior of steel in organic acids is an important problem for the industries of organic chemistry and petrochemistry. In respect of this problem, Heitz et al. ${ }^{1) \sim 4)}$ have been reported by polarization curve and weight loss test about many systems for metals and solvents. We have been also reported by means of an impedance analysis on the anodic dissolution of pure iron in nonaqueous formic acid. ${ }^{5), 6)}$ However, thus far the influence of water in organic acids on the corrosion of metals has not been clarified and, further, there is no analysis by impedance measurement at the stainless steel-organic acid interface.

In this work, formic acid was chosen as an organic acid, because it consists of the simplest structure in organic acids, and is a high corrosive solvent. As a test steel, we chose Type 304 SS which has a high corrosion-resistance and is widely used in general apparatus. The corrosion behavior of Type 304 SS in formic acid solution was investigated in detail by measuring the corrosion weight loss, the polarization curve, and the impedance at the steel-formic acid interface. Especially, the importance of this work is to elucidate the influence of water content against corrosion rate.

In the case of weight loss test, the measurement

* 2641, Higashi-kameyama, Yamazaki, Noda, Chiba 278 Japan was made at both room temperature and boiling point to clarify the influence of temperature as a parameter of corrosion rate. Further, the possibility of the selective corrosion dissolution in this system was examined by an atomic absorption analysis about $\mathrm{Fe}, \mathrm{Cr}$ and $\mathrm{Ni}$ dissolved in the solution after weight loss test at boiling point.

\section{Experimental}

\section{1 Materials}

The chemical components of specimen and electrode of Type $304 \mathrm{SS}$ are shown in Table 1. The specimens were annealed in vacuo at $1040^{\circ} \mathrm{C}$ for $40 \mathrm{~min}$ and water-quenched. Before each test, the specimen was abraded with emery papers up to 1200 grades, and then degreased with acetone in a supersonic washer.

The reagent grade formic acid $(98 \sim 99 \%)$ was refluxed after addition of boron trioxide $\left(\mathrm{B}_{2} \mathrm{O}_{3}\right)$, and then distilled under reduced pressure (ca. 70 Torr). The potassium formate as a supporting electrolyte was recrystallized, then rinsed with ethanol, and dried under reduced pressure. The concentration of formic acid was changed on $\mathbf{0}$ 99.8 vol. \%. When the water content in the solu-

Table 1 Chemical compositions of test materials (Type 304 SS) (wt. \%).

\begin{tabular}{c|c|c|c|c|c|c}
\hline \hline $\mathrm{C}$ & $\mathrm{Si}$ & $\mathrm{Mn}$ & $\mathrm{P}$ & $\mathrm{S}$ & $\mathrm{Ni}$ & $\mathrm{Cr}$ \\
\hline 0.07 & 0.51 & 1.23 & 0.032 & 0.006 & 8.49 & 18.22 \\
\hline
\end{tabular}


tion was less than $1 \%$, the trace amounts of water were exactly determined using the method of Karl Fischer.

\section{2 Weight loss measurements}

The size of specimen was $20 \times 50 \times 0.5 \mathrm{~mm}$. The concentration of formic acid used was in the range of $0 \sim 99.8 \%$. Test specimen was examined in the solution at boiling point for 24 hours. Measurement at room temperature $\left(30^{\circ} \mathrm{C}\right)$ was carried out for 33 days. About $430 \mathrm{ml}$ of solution was used in each run.

\section{3 Polarization measurements}

The specimen of $15 \times 150 \times 0.5 \mathrm{~mm}$ was covered with a silicon rubber sealant except both-sided surface of $3 \mathrm{~cm}^{2}$. The counter electrode was a platinum plate of $30 \times 40 \times 0.5 \mathrm{~mm}$. The potential was measured with reference to a saturated calomel electrode (SCE). The concentration of formic acid was changed on $1 \sim 99.5 \%$. Potassium formate was added in the solution $(0.5 \mathrm{M})$. The solutions were deaerated with purified nitrogen gas for 2 hours, and the exchange of solution was made by using the pressure of purified nitrogen gas. Prior to measurements, the working electrode was reduced at $-10 \mathrm{~mA} / \mathrm{cm}^{2}$ for $30 \mathrm{~min}$, and then the solution was exchanged. The polarization measurement in steady state was first carried out by polarizing from $E_{\text {corr }}$ to cathodic potential of $300 \sim 400 \mathrm{mV}$ with the potential step method $(25 \mathrm{mV} / 30 \mathrm{sec})$, and then polarized to the anodic direction through $E_{\text {corr }}$ until a current peak in active region appears.

\section{4 Impedance measurements}

The test electrode of $2 \mathrm{~mm}$ diameter is made up of a cylindrical cross section whose the lateral surface is covered by Teflon. The counter electrode was a platinum spiral wire. The treatments of the solution and the electrode were the same as those of polarization measurements. After the constant current reduction, the electrode was permitted to stand for at least 1.5 hour until the $E_{\text {corr }}$ stabilized. The impedance of electrode was measured at $E_{\text {corr }}$. In the frequency range $2 \mathrm{~K}-1 \mathrm{~Hz}$, a Lock-in amplifier was used in conjunction with a potentiostat, and in the frequency range $1 \sim 0.004 \mathrm{~Hz}$, Lissajou's figures were recorded on $\mathrm{X}-\mathrm{Y}$ recorder. The reference signal of sinusoidal wave was employed within $3.5 \mathrm{mV}$ (RMS).

\subsection{Atomic absorption analysis}

In order to analyze each element dissolved in solution, air-acetylene flame was used, and the wave length of hollow-cathode lamps was employed
$2483 \AA$ for $\mathrm{Fe}, 3579 \AA$ for $\mathrm{Cr}$ and $2320 \AA$ for $\mathrm{Ni}$, respectively.

\section{Results and Discussion}

\section{1 Corrosion weight loss}

The experimental results at room temperature are shown in Table 2. The weight loss was little observed on solutions ranging in concentration from 5 to $70 \%$ of formic acid. The highest value of corrosion rate was $0.32 \mathrm{mdd}$. When the specimens were taken out of the solution, the surfaces of any test pieces used were scarcely altered as before immersion, and maintained a silvery metallic luster.

Figure 1 shows the result of weight loss at boiling point $\left(95 \sim 102^{\circ} \mathrm{C}\right)$. In such cases, the corrosion rate increased with the concentration of formic acid, and a maximum value was $470 \mathrm{mdd}$ $(2.1 \mathrm{~mm} / \mathrm{yr})$ in a concentration of $70 \%$. However, in concentration over $70 \%$, the corrosion rate decreased inversely. For these reasons, it would be ascribed to lack water whose protons $\left(\mathrm{H}^{+}\right)$as an attacker are necessary to stabilize the hydronium ions $\left(\mathrm{H}_{3} \mathrm{O}^{+}\right)$. Namely, since the dissociation of formic acid decreases with increasing concentration, the corrosion rate would be small in spite of the presence of the large amounts of formic acid over $70 \%$. In brief, it is considered that the concentration of formic acid of ca. $70 \%$ represents a boundary between the increase and decrease of corrosion rate. This may be correspond to that the electric conductivity of various solutions exhibits a maximum value with increasing concen-

Table 2 Corrosion weight loss in $\mathrm{HCOOH}$ solution at $30^{\circ} \mathrm{C}$.

\begin{tabular}{c|c|c|c|c|c}
\hline \hline $\mathrm{HCOOH}(\%)$ & 5 & 10 & 20 & 50 & 70 \\
\hline $\mathrm{mg}$ & 0.9 & 1.9 & 2.2 & 1.5 & 0.3 \\
\hline $\mathrm{mdd}$ & 0.13 & 0.27 & 0.32 & 0.22 & 0.04 \\
\hline
\end{tabular}

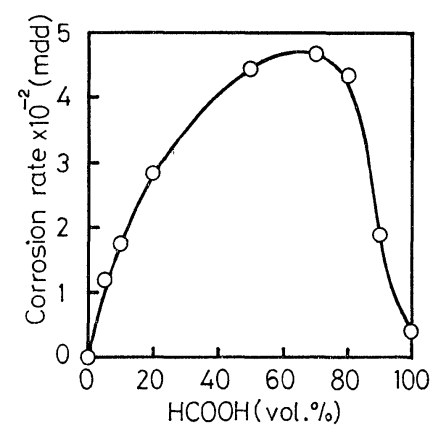

Fig. 1 Corrosion weight loss vs. concentration of $\mathrm{HCOOH}$ at boiling point. 


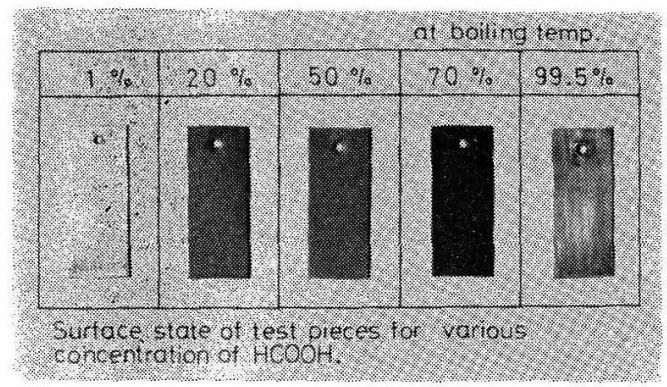

Fig. 2 Surface state of test pieces for various concentrations of $\mathrm{HCOOH}$.

tration of electrolyte.

Figure 2 shows the photograph of test pieces after weight loss test at boiling point. The surfaces in concentrations of $1 \%$ and $99.5 \%$ of formic acid are little changed. But with increasing concentration from $20 \%$ to $70 \%$, the surface of test piece became rough gradually, and brown particles which are regarded as corrosion products were spread on the whole surface of the test piece. These particles seemed to be hydroxides of metals, especially iron, as can be seen from the color. Consequently, in order to clarify the composition of these particles, they were analyzed by the IR method (MIR 41 type) of a reflection type after the test piece was dried under reduced pressure. However, since the specific absorption spectra were not observed, the corrosion products could not be analyzed by this method. It was considered that in this connection the test piece should be analyzed in the state of immersion in the solution, i.e., in situ.

Figurc 3 shows the micrograph of the test piece after weight loss test at both room temperature and boiling point. At room temperature, the lateral striped pattern was the polished streak by emery paper, and it was little changed in spite of the immersion for 33 days. On the other hand, such streaks at boiling point disappeared completely by boiling for 24 hours. Fontana et al. ${ }^{7)}$ have been described that Type 304 SS is resistant to corrosion in a formic acid solution of $80 \%$ at $24^{\circ} \mathrm{C}$, but is nonresistant to corrosion at $100^{\circ} \mathrm{C}$.

As a result of the weight loss test in the present work, the corrosion-resistance of Type 304 SS against formic acid of any concentration examined was sufficiently high at $30^{\circ} \mathrm{C}$ of room temperature, and became low remarkably at boiling point.

\section{2 Polarization characteristics}

Main polarization curves measured in deaerated formic acid solution at room temperature are shown in Fig. 4. The Tafel linear region was

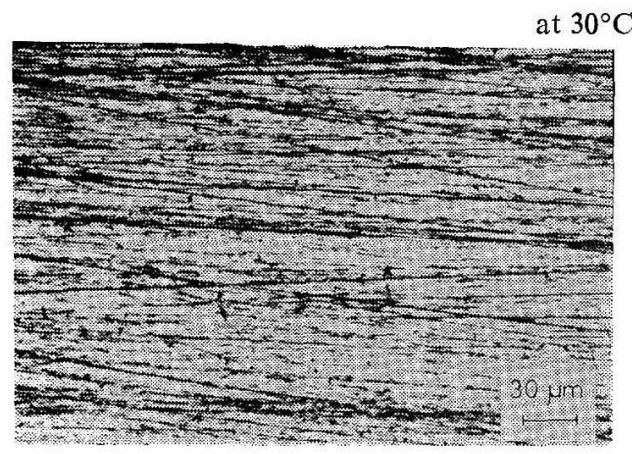

at b.p.

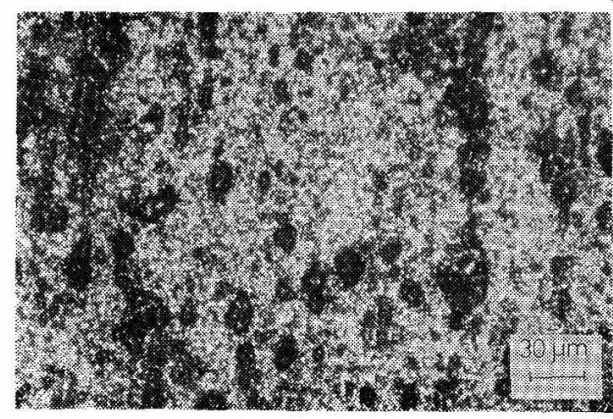

Fig. 3 Micrograph of the test piece after corrosion weight loss at room temperature $\left(30^{\circ} \mathrm{C}\right)$ and boiling point $\left(95 \sim 102^{\circ} \mathrm{C}\right)$.

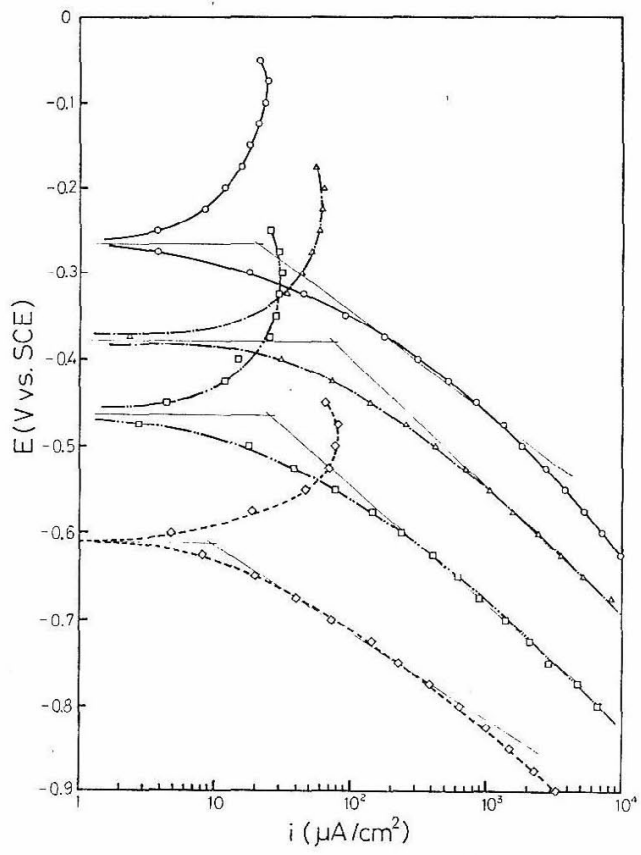

Fig. 4 Polarization curves in deaerated $\mathrm{HCOOH}$ $+0.5 \mathrm{M} \mathrm{HCOOK}$ aqueous solution at $25^{\circ} \mathrm{C}$ - - - $99.5 \% \mathrm{HCOOH},--\triangle---: 70.0 \%$ $\mathrm{HCOOH}, \ldots-\square-\ldots: 20.0 \% \mathrm{HCOOH}$, $\ldots-\ldots: 1.00 \% \mathrm{HCOOH}$. 
observed on the cathodic curve, but it was not observed on the anodic curve. Therefore the corrosion current density $\left(i_{\text {corr }}\right)$ was determined by extrapolation of the cathodic Tafel line to the corrosion potential $\left(E_{\text {eorr }}\right)$.

The change of the $i_{\text {corr }}$ vs. the concentration of formic acid is shown in Fig. 5. A maximum value of the corrosion rate $\left(i_{\text {corr }}\right)$ was observed in a concentration of $70 \%$ of formic acid as well as the weight loss test at boiling point. But this result was not consistent with that obtained by the weight loss test at room temperature. For these reasons, it would be explained that the weight loss was too small to be reliable because the corrosion rate at room temperature was remarkably small.

The relation between the $\mathrm{pH}$ of each solution and $E_{\text {corr }}$ is summarized in Table 3. When the concentration of formic acid was over $80 \%$, the solution was almost nonaqueous state. Consequently, the $\mathrm{pH}$ of the solution was omitted be-

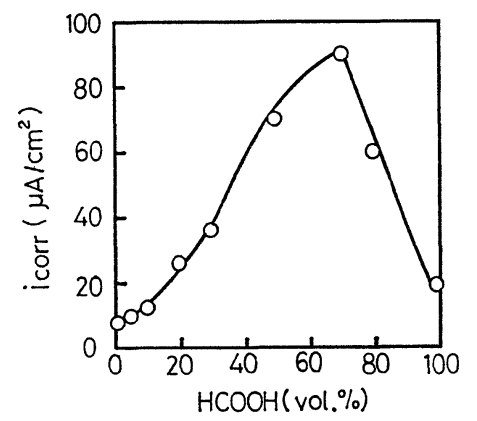

Fig. 5 Effect of the concentration of $\mathrm{HCOOH}$ on the corrosion current density at $25^{\circ} \mathrm{C}$.

Table $3 \mathrm{pH}$ and $E_{\text {corr }}$ vs. concentration of $\mathrm{HCOOH}$.

\begin{tabular}{c|c|c|c|c|c|c|c}
\hline \hline $\mathrm{HCOOH}(0)$ & 1 & 5 & 10 & 20 & 30 & 50 & 70 \\
\hline $\mathrm{pH}$ & 3.86 & 3.00 & 2.60 & 2.15 & 1.80 & 0.86 & 0.14 \\
\hline $\begin{array}{c}\text { Ecorr } \\
(\mathrm{mV} \text { vs. SCE) }\end{array}$ & -607 & -525 & -520 & -470 & -470 & -430 & -373 \\
\hline
\end{tabular}

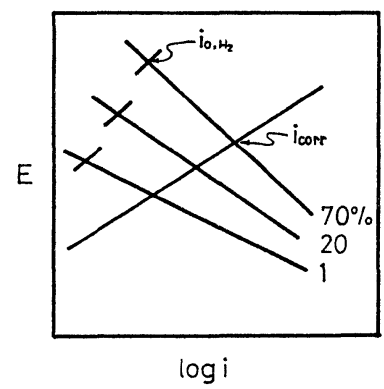

Fig. 6 Conception diagram of the change of $E_{\text {corr }}$ with $\mathrm{pH}$. cause it was impossible to measure by usual $\mathrm{pH}$ measurement. In this case, the $\mathrm{pH}$ became small with increasing concentration of formic acid, and the $E_{\text {corr }}$ was shifted to more noble potential.

The change of $E_{\text {corr }}$ with $\mathrm{pH}$ is explained by a conception diagram as Fig. 6. The corrosion reaction consists of the anodic dissolution reaction of Type 304 SS and the hydrogen evolution reaction because the solution is deaerated in this work. Therefore the change of the $\mathrm{pH}$ has an influence upon the equilibrium potential $\left(E_{\text {eq }}\right)$ of the hydrogen evolution reaction, and the $E_{\text {eq }}$ shifts to more noble potential with decreasing $\mathrm{pH}$. Further the $E_{\text {eq }}$ is calculated by Nernst's equation from the $\mathrm{pH}$ of various solutions, and the exchange current density $\left(i_{\mathrm{O}_{\mathrm{H}}}\right)$ of the hydrogen electrode reaction is determined by extrapolation of the cathodic polarization curve to $E_{\text {eq }}$. Consequently, the maximum $i_{\mathrm{O}, \mathrm{H}_{2}}$ is obtained in a concentration of $70 \%$ of formic acid solution. Hence the intersection on the anodic polarization curve $\left(i_{\text {corr }}\right)$ also increases, and the corrosion reaction proceeds. As these results, the $E_{\text {corr }}$ also shifts to more noble potential with increasing concentration of formic acid.

The cathodic Tafel slope $\left(b_{\text {eathodic }}\right)$ of the polarization curve obtained is $-120 \mathrm{mV} /$ decade, i.e., $-2.303 \times 2 R T / F$. Since the cathodic reaction is considered as the hydrogen evolution reaction, the

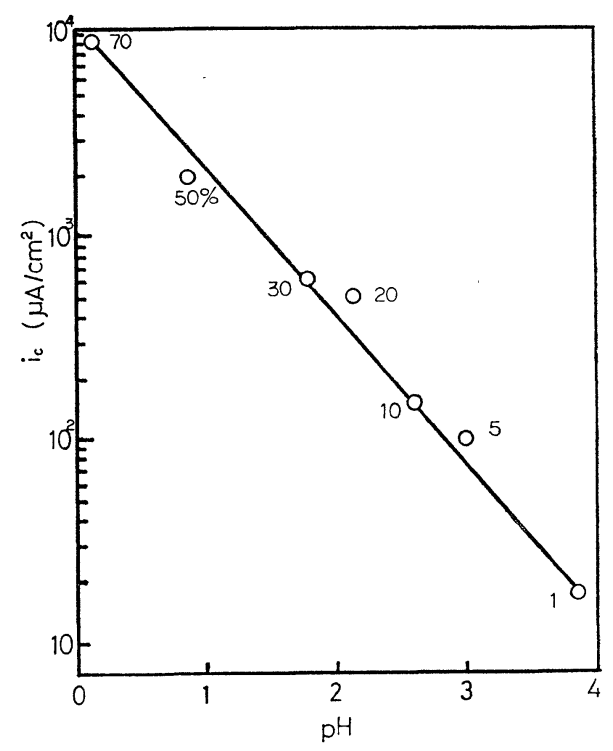

Fig. 7 Cathodic current density of Type 304 SS electrode as function of $\mathrm{pH}$ at constant potential $(E=-0.650 \mathrm{~V}$ vs. SCE) in deaerated $\mathrm{HCOOH}+0.5 \mathrm{M} \mathrm{HCOOK}$ aqueous solution at $25^{\circ} \mathrm{C}$. 
partial cathodic current density $\left(i_{\mathrm{c}}\right)$ can be expressed by Eq. (1).

$$
\left|i_{\mathrm{c}}\right|=k_{\mathrm{c}} F a_{\left(\mathrm{H}^{+},\right.}^{n^{+}} \exp (-F E / 2 R T)
$$

where $k_{\mathrm{c}}$ is the cathodic reaction rate constant, $F$ the Faraday constant, $a_{\left(\mathrm{H}^{+}\right)}$the proton activity, and $n$ the reaction order of proton. According to Eq. [1], the reaction order is given by Eq. (2).

$$
n=\left(\partial \log i_{\mathrm{c}} / \partial \mathrm{pH}\right)_{E}
$$

In accordance with this equation, the plot of $\log$ $i_{\mathrm{c}}$ vs. $\mathrm{pH}$ was found to be linear by using the current densities observed at a constant potential of $-0.650 \mathrm{~V}$ as is shown in Fig. 7. These plots were made in the concentration range from $1 \%$ to $70 \%$ of formic acid which the $\mathrm{pH}$ could be measured. From this figure, the slope $(n)$ was -0.725 . Consequently, it is considered that the hydrogen evolution reaction was approximately first order with respect to the activity of proton.

The relationship between $E_{\text {corr }}$ and $\mathrm{pH}$ is shown in Fig. 8. A plot of $E_{\text {corr }}$ vs. pH can be seen to give a straight line having a slope $\left(=d E_{\text {corr }} / d \mathrm{pH}\right)$ of $-0.062 \mathrm{~V} / \mathrm{pH}$, i.e., $-2.303 \times(R T / F)$. Therefore

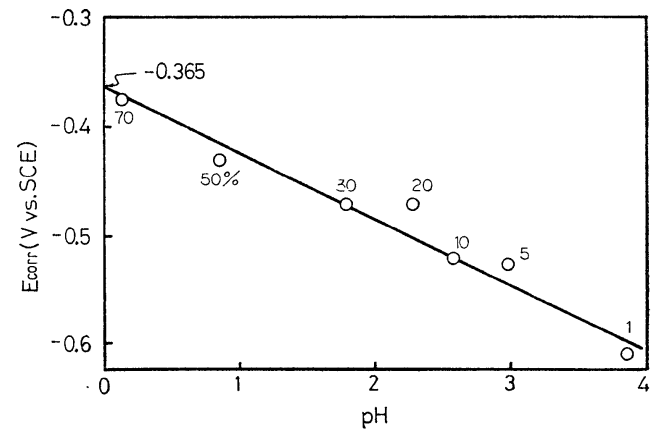

Fig. 8 Relationship between the corrosion potential of Type $304 \mathrm{SS}$ and the $\mathrm{pH}$ in deaerated $\mathrm{HCOOH}+0.5 \mathrm{M} \mathrm{HCOOK}$ aqueous solution at $25^{\circ} \mathrm{C}$.

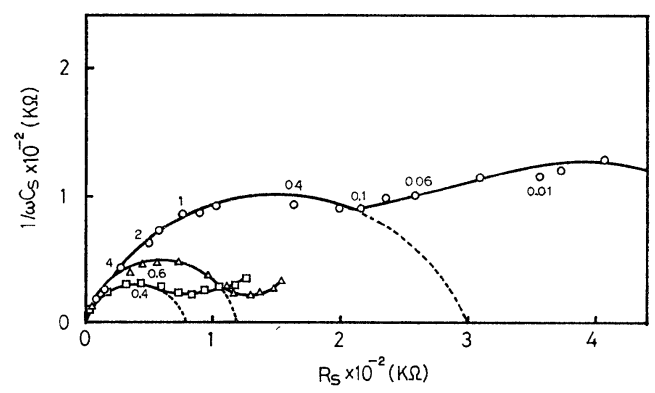

Fig. 9 Complex impedance plane plot in Type $304 \mathrm{SS} / \mathrm{HCOOH}+0.5 \mathrm{~m} \mathrm{HCOOK}$ aqueous solution at $E_{\text {corr }}$ (Frequencies in $\mathrm{Hz}$ ).

——: $99.5 \% \mathrm{HCOOH},-\triangle-: 80.0 \%$ $\mathrm{HCOOH},-\square-: 50.0 \% \mathrm{HCOOH}$.
Table $4 \theta$ and $C_{\mathrm{d} 1}$ obtained from impedance measurements.

\begin{tabular}{c|r|r|r|r}
\hline \hline $\mathrm{HCOOH}(\%)$ & 20 & 50 & 80 & 99.5 \\
\hline$\theta(\mathrm{k} \Omega)$ & 80 & 80 & 120 & 300 \\
\hline $\mathrm{C}_{\mathrm{d} 1}\left(\mu \mathrm{F} / \mathrm{cm}^{2}\right)$ & 158 & 158 & 70 & 42 \\
\hline
\end{tabular}

the equation of the straight line is given by Eq. (3).

$$
\begin{aligned}
E_{\text {corr }} & =-0.062 \mathrm{pH}-0.365 \\
& =-2.303(R T / F) \mathrm{pH}-0.365
\end{aligned}
$$

As a result, it was recognized that $E_{\text {corr }}$ is linearly dependent on the $\mathrm{pH}$ of solution in the concentration range up to at least $70 \%$ of formic acid.

\section{3 Impedance measurements}

The complex impedance plane plots are shown in Fig. 9. The loci of plots in the high frequency region at various concentrations showed a part of the semicircle. In this case, the center of the semicircle was somewhat deviated from the real axis. In the low frequency region, it showed a part of capacitive circular arc. Such a capacitive arc seems to be suggested the presence of adsorption intermediates on the electrode surface.

The charge transfer resistance or the corrosion reaction resistance $(\theta)$ was determined from a diameter of an arc in the high frequency region, and the electric double layer capacity $\left(C_{\mathrm{d} 1}\right)$ was determined from an angular frequency $\left(\omega_{\max }\right)$ of a vertex of the arc and $\theta$. The values of $\theta$ and $C_{\mathrm{d} 1}$ at each concentration are shown in Table 4. The $\theta$ became small with increasing amounts of water, whereas the $C_{\mathrm{d} 1}$ became large. It would be qualitatively explained that the increase of $C_{\mathrm{a} 1}$ is based on the increase of surface area because the electrode surface becomes rough as the corrosion reaction proceeds ${ }^{8)}$.

From the result of impedance measurement, the concentration of formic acid showing the highest corrosion rate was not determined explicitly. However, it was found that the corrosion of Type 304 SS is fairly stimulated by containing the large amounts of water in formic acid.

\section{4 Selective corrosion dissolution}

The proportions of elution of $\mathrm{Fe}, \mathrm{Cr}$ and $\mathrm{Ni}$ in various formic acid solutions by an atomic absorption method are shown in Fig. 10. The sum of these proportions was assumed as $97 \%$ from its components. The proportions in each solution became about $70 \%$ for $\mathrm{Fe}, 18 \%$ for $\mathrm{Cr}$ and $9 \%$ for $\mathrm{Ni}$, respectively. Consequently, the selective corrosion dissolution was not observed because these proportions were approximately the same as that of the components of Type 304 SS. 


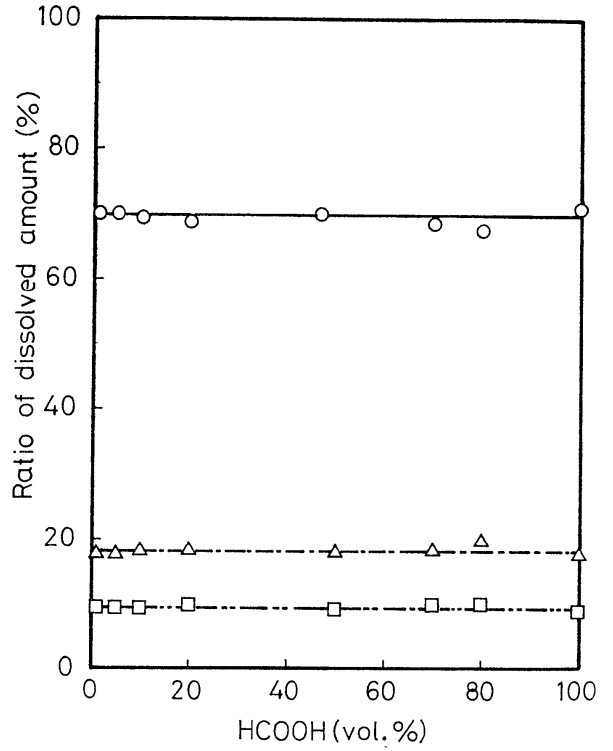

Fig. 10 Ratio of dissolved amounts of each element vs. concentration of $\mathrm{HCOOH}$ after weight loss at boiling point.

$-\mathrm{O}-\mathrm{Fe},---\Delta---: \mathrm{Cr},---\square----: \mathrm{Ni}$.

\section{Conclusions}

The results investigated about the corrosion behavior of Type 304 SS in formic acid solution are as follows:

(1) The corrosion rate of the steel was changed with the remarkable dependence on the concentration of formic acid, and a maximal rate was observed when the concentration was $70 \%$.

(2) The corrosion rate was changed with the remarkable dependence on the temperature, and the rate at boiling point was several thousand times greater than that at room temperature.

(3) The cathodic current density $\left(i_{c}\right)$ was given as follows:

$$
\left|i_{\mathrm{c}}\right|=k_{\mathrm{c}} F a_{\left(\mathrm{H}^{+}\right)}^{n^{+}} \exp (-F E / 2 R T)
$$

where the reaction order of proton $(n)$ became about one.

(4) The relationship between $E_{\text {corr }}$ and $\mathrm{pH}$ was shown by a straight line, and the equation was formulated as follows:

$$
E_{\text {eorr }}=-2.303(R T / F) \mathrm{pH}-0.365
$$

(5) In the complex impedance plane plots, the appearance of the loci of capacitive circular arc was suggested the presence of adsorption intermediates on the electrode surface.

(6) The selective corrosion dissolution about $\mathrm{Fe}, \mathrm{Cr}$ and $\mathrm{Ni}$ was not recognized.

(Received December 5, 1981)

\section{References}

1) E. Heitz: Werks. u. Korros., 21, 360 (1970).

2) E. Heitz: "Corrosion of Metals in Organic Solvents", Advanced in Corrosion Science and Technology, Ed. by M. G. Fontana and R. W. Staehle, Vol. 4, p. 149 Plenum Press (1974).

3) E. Constantinescu and E. Heitz: Corros. Sci., 16, 857 (1976).

4) E. Heitz and C. Kyriazis: Ind. Eng. Chem. Prod. Res. Dev., 17, 37 (1978).

5) I. Sekine, H. Ohkawa and T. Handa: Boshoku Gijutsu, 30, 634 (1981).

6) I. Sekine, H. Ohkawa and T. Handa: Corros. Sci., to be submitted.

7) M. G. Fontana and N. D. Greene: "Corrosion Engineering", p. 263, McGraw-Hill (1967).

8) S. Haruyama, T. Tsuru and M. Anan: Boshoku Gijutsu, 27, 449 (1978). 